\title{
Harmonic starlikeness and convexity of integral operators generated by Poisson distribution series
}

\author{
Saurabh Porwal and Divesh SRIVastava
}

\begin{abstract}
The purpose of the present paper is to establish connections between various subclasses of harmonic univalent functions by applying certain integral operator involving the Poisson distribution series. To be more precise, we investigate such connections with harmonic starlike and harmonic convex mappings in the plane.
\end{abstract}

\section{INTRODUCTION}

Let $A$ denote the class of functions $f(z)$ of the form

$$
f(z)=z+\sum_{n=2}^{\infty} a_{n} z^{n}
$$

which are analytic in the open unit disc $U=\{z: z \in C$ and $|z|<1\}$ and satisfy the normalization condition $f(0)=f^{\prime}(0)-1=0$. Further, we denote by $S$ the subclass of $A$ consisting of functions of the form (1) which are also univalent in $U$. A continuous complex-valued function $f=u+i v$ is said to be harmonic in a simply-connected domain $D$ if both $u$ and $v$ are real harmonic in $D$. In any simply-connected domain we can write $f=h+\bar{g}$, where $h$ and $g$ are analytic in $D$. We call $h$ the analytic part and $g$ the coanalytic part of $f$. A necessary and sufficient condition for $f$ to be locally univalent and sense-preserving in $D$ is that $\left|h^{\prime}(z)\right|>\left|g^{\prime}(z)\right|, z \in D$. See Clunie and Sheil-Small [4], for more basic results on harmonic functions one may refer to the following standard introductory text book by Duren [6], (see also [1]).

Let $H$ be the family of all harmonic functions of the form $f=h+\bar{g}$, where

$$
h(z)=z+\sum_{n=2}^{\infty} A_{n} z^{n}, g(z)=\sum_{n=1}^{\infty} B_{n} z^{n},\left|B_{1}\right|<1 .
$$

2000 Mathematics Subject Classification. Primary: 30C45.

Key words and phrases. Harmonic, Univalent functions, Poisson Distribution Series. 
Denote by $S_{H}$ the subclass of $H$ functions $f=h+\bar{g}$ that are harmonic univalent and sense-preserving in the open unit disk $U=\{z:|z|<1\}$ for which $f(0)=f_{z}(0)-1=0$.

Note that $S_{H}$ reduces to class $S$ of normalized analytic univalent functions if the co-analytic part of its member is zero. In fact, Clunie and Sheil-Small [4] investigated the class $S_{H}$. We also let the subclass $S_{H}^{0}$ of $S_{H}$

$$
S_{H}^{0}=\left\{f=h+\bar{g} \in S_{H}: g^{\prime}(0)=B_{1}=0\right\} .
$$

The classes $S_{H}^{0}$ and $S_{H}$ were first studied in [4].

A function $f(z)$ of the form (2) in $S_{H}$ is said to be harmonic starlike of order $\alpha,(0 \leq \alpha<1)$ in $U$, if and only if

$$
\frac{\partial}{\partial \theta}\{\arg f(z)\}>\alpha, z \in U
$$

and is said to be harmonic convex of order $\alpha,(0 \leq \alpha<1)$ in $U$, if and only if

$$
\frac{\partial}{\partial \theta}\left\{\arg \left(\frac{\partial}{\partial \theta} f(z)\right)\right\}>\alpha, z \in U
$$

The classes of all harmonic starlike functions of order $\alpha$ and harmonic convex functions of order $\alpha$ are denoted by $S_{H}^{*}(\alpha)$ and $K_{H}(\alpha)$, respectively. These classes have been extensively studied by Jahangiri [8].

For $\alpha=0$, these classes $S_{H}^{*}(\alpha)$ and $K_{H}(\alpha)$ were denoted by $S_{H}^{*}$ and $K_{H}$ respectively. These classes were studied in detail by Silverman [16] and Silverman and Silvia [17], (see also [3]). Further, we let $K_{H}^{0}, S_{H}^{*, 0}$ and $C_{H}^{0}$ denote the subclasses of $S_{H}^{0}$ of harmonic functions which are, respectively, convex, starlike and close-to-convex in $U$. For definitions and properties of these classes, one may refer to $([1],[4])$ or $[6]$.

Very recently, Porwal [12] introduce a power series whose coefficients are probabilities of Poisson distribution

$$
K(m, z)=z+\sum_{n=2}^{\infty} \frac{m^{n-1}}{(n-1) !} e^{-m} z^{n} .
$$

By ratio test the radius of convergence of above series is infinity. Using the above series they obtain some interesting results on certain classes of analytic univalent functions. Some other interesting results also found in [5], [9] and [10], (see also [7], [11]).

The convolution (or Hadamard product) of two series $f(z)=\sum_{n=0}^{\infty} a_{n} z^{n}$ and $g(z)=\sum_{n=0}^{\infty} b_{n} z^{n}$ is defined as the power series

$$
(f * g)(z)=\sum_{n=0}^{\infty} a_{n} b_{n} z^{n} .
$$

Using the definition (5), we introduce the integral operator $I: H \rightarrow H$ by 


$$
I(f) \equiv I\left(m_{1}, m_{2}\right) f(z)=H(z)+\overline{G(z)},
$$

where

$$
H(z)=h(z) * \int_{0}^{z} \frac{K\left(m_{1}, t\right)}{t} d t, G(z)=g(z) * \int_{0}^{z} \frac{K\left(m_{2}, t\right)}{t} d t,
$$

or equivalently

$$
\text { (7) } H(z)=z+\sum_{n=2}^{\infty} \frac{e^{-m_{1}} m_{1}^{n-1}}{n !} A_{n} z^{n}, G(z)=B_{1} z+\sum_{n=2}^{\infty} \frac{e^{-m_{2}} m_{2}^{n-1}}{n !} B_{n} z^{n} \text {, }
$$

where $*$ denotes the usual Hadamard product or convolution of two power series. The hypergeometric series plays an important role in Geometric Function theory. Recently Ahuja [2] studied the harmonic starlikeness and convexity of integral operators generated by hypergeometric series. Analogues to these results Porwal [13],(see also [14]-[15], [18]), studied the harmonic starlikeness and convexity of integral operators generated by generalized Bessel functions. In the present paper motivated with the above mentioned work we establish a number of connections between the classes $S_{H}^{*}(\alpha), K_{H}(\alpha), K_{H}^{0}, S_{H}^{*, 0}, C_{H}^{0}$ by applying the integral operator $I$.

\section{Preliminary Lemmas}

To prove our main results we shall require the following lemmas.

Lemma 2.1. ([6]) If $f=h+\bar{g} \in K_{H}^{0}$ where $h$ and $g$ are given by (2) with $B_{1}=0$, then

$$
\left|A_{n}\right| \leq \frac{n+1}{2},\left|B_{n}\right| \leq \frac{n-1}{2} .
$$

Lemma 2.2. ([8]) Let $f=h+\bar{g}$ be given by (2). If for some $\alpha(0 \leq \alpha<1)$ and the inequality

$$
\sum_{n=2}^{\infty}(n-\alpha)\left|A_{n}\right|+\sum_{n=1}^{\infty}(n+\alpha)\left|B_{n}\right| \leq 1-\alpha,
$$

is satisfied, then $f$ is harmonic, sense-preserving, univalent functions in $U$ and $f \in S_{H}^{*}(\alpha)$.

Define $T S_{H}^{*}(\alpha)=S_{H}^{*}(\alpha) \cap T$ and $T K_{H}(\alpha)=K_{H}(\alpha) \cap T$, where $T$ consists of the functions $f=h+\bar{g}$ in $S_{H}$ so that $h(z)$ and $g(z)$ are of the form

$$
h(z)=z-\sum_{n=2}^{\infty}\left|A_{n}\right| z^{n}, g(z)=\sum_{n=1}^{\infty}\left|B_{n}\right| z^{n},\left|B_{1}\right|<1 .
$$

Remark 2.1. In [8], it is also shown that $f=h+\bar{g}$ given by (9) is in the family $T S_{H}^{*}(\alpha)$, if and only if the coefficient condition (8) holds. Moreover, if $f \in T S_{H}^{*}(\alpha)$, then

$$
\left|A_{n}\right| \leq \frac{1-\alpha}{n-\alpha}, n \geq 2,\left|B_{n}\right| \leq \frac{1-\alpha}{n+\alpha}, n \geq 1 .
$$


Lemma 2.3. ([8]) Let $f=h+\bar{g}$ be given by (2). If for some $\alpha(0 \leq \alpha<1)$ and the inequality

$$
\sum_{n=2}^{\infty} n(n-\alpha)\left|A_{n}\right|+\sum_{n=1}^{\infty} n(n+\alpha)\left|B_{n}\right| \leq 1-\alpha,
$$

is satisfied, then $f$ is harmonic, sense-preserving univalent functions in $U$ and $f \in K_{H}(\alpha)$.

Remark 2.2. In [8], it is also shown that $f=h+\bar{g}$ given by (9) is in the family $T K_{H}(\alpha)$, if and only if the coefficient condition (11) holds. Moreover, if $f \in T K_{H}(\alpha)$, then

$$
\left|A_{n}\right| \leq \frac{1-\alpha}{n(n-\alpha)}, n \geq 2,\left|B_{n}\right| \leq \frac{1-\alpha}{n(n+\alpha)}, n \geq 1 .
$$

Lemma 2.4. ([6]) Let $f=h+\bar{g} \in S_{H}^{*, 0}$ or $C_{H}^{0}$ where $h$ and $g$ are given by (2) with $B_{1}=0$, then

$$
\left|A_{n}\right| \leq \frac{(2 n+1)(n+1)}{6},\left|B_{n}\right| \leq \frac{(2 n-1)(n-1)}{6}, n \geq 2 .
$$

\section{Main Results}

In our first result, we determine conditions which guarantee that the integral operator $I$ is harmonic starlike in $U$.

Theorem 3.1. If $0 \leq \alpha<1, m_{j}>0$ for $j=1,2$. Also, suppose $f=h+\bar{g} \in$ $H$ is given by (2). If the inequalities

$$
\begin{aligned}
& \text { (i) } \sum_{n=2}^{\infty}\left|A_{n}\right|+\sum_{n=1}^{\infty}\left|B_{n}\right| \leq 1,\left|B_{1}\right|<1 \\
& \text { (ii) } e^{-m_{1}}+e^{-m_{2}} \geq 1+\left|B_{1}\right|
\end{aligned}
$$

are satisfied, then the integral operator $I$ is sense-preserving, harmonic univalent and maps $H$ in to $S_{H}^{*}$.

Proof. Note that

$$
I\left(m_{1}, m_{2}\right) f(z)=H(z)+\overline{G(z)},
$$

where $H(z)$ and $G(z)$ are given by (7). In order to show that $I$ is locally univalent and sense-preserving it suffices to show that $\left|H^{\prime}(z)\right|-\left|G^{\prime}(z)\right|>0$ in $U$. Using the condition (i), we have

$$
\begin{aligned}
\left|H^{\prime}(z)\right| & -\left|G^{\prime}(z)\right| \\
& >1-\sum_{n=2}^{\infty} n \frac{e^{-m_{1}} m_{1}^{n-1}}{n !}-\sum_{n=2}^{\infty} n \frac{e^{-m_{2}} m_{2}^{n-1}}{n !}-\left|B_{1}\right| \\
& =1-\left|B_{1}\right|-e^{-m_{1}}\left(e^{m_{1}}-1\right)-e^{-m_{2}}\left(e^{m_{2}}-1\right) \\
& =1-\left|B_{1}\right|-\left(1-e^{-m_{1}}\right)-\left(1-e^{-m_{2}}\right)
\end{aligned}
$$




$$
\begin{aligned}
& =e^{-m_{1}}+e^{-m_{2}}-1-\left|B_{1}\right| \\
& \geq 0, \text { from (ii). }
\end{aligned}
$$

To show that $I(f)$ is univalent in $U$, we follow the method of Theorem 1 in [8]. That is, for $z_{1} \neq z_{2}$ in $U$, it suffices to prove that

$$
\Re \frac{f\left(z_{2}\right)-f\left(z_{1}\right)}{z_{2}-z_{1}}>\int_{0}^{1}\left(\Re H^{\prime}(z(t))-\left|G^{\prime}(z(t))\right|\right) d t .
$$

Since from the given condition (i), we have

$$
\Re H^{\prime}(z)-\left|G^{\prime}(z)\right|>1-\sum_{n=2}^{\infty} n \frac{e^{-m_{1}} m_{1}^{n-1}}{n !}-\left|B_{1}\right|-\sum_{n=2}^{\infty} n \frac{e^{-m_{2}} m_{2}^{n-1}}{n !}
$$

it follows from the given hypothesis that the last inequality is positive. Therefore, from the inequality (13) we have

$$
\Re \frac{f\left(z_{2}\right)-f\left(z_{1}\right)}{z_{2}-z_{1}}>0 .
$$

This proves the univalence of $I(f)$.

In order to prove that $I(f) \in S_{H}^{*} \equiv S_{H}^{*}(0)$, it suffices to show that $P_{1} \leq 1$, because of Lemma 2.2, where

$$
P_{1}=\sum_{n=2}^{\infty} n \frac{e^{-m_{1}} m_{1}^{n-1}}{n !}\left|A_{n}\right|+\left|B_{1}\right|+\sum_{n=2}^{\infty} n \frac{e^{-m_{2}} m_{2}^{n-1}}{n !}\left|B_{n}\right| .
$$

Since $\left|A_{n}\right| \leq 1,\left|B_{n}\right| \leq 1, \forall n \geq 2$, because of given condition (i), we obtain

$$
\begin{aligned}
P_{1} & \leq \sum_{n=2}^{\infty} \frac{e^{-m_{1}} m_{1}^{n-1}}{(n-1) !}+\left|B_{1}\right|+\sum_{n=2}^{\infty} \frac{e^{-m_{2}} m_{2}^{n-1}}{(n-1) !} \\
& =\left(1-e^{-m_{1}}\right)+\left|B_{1}\right|+\left(1-e^{-m_{2}}\right) \\
& \leq 1, \text { from (ii). }
\end{aligned}
$$

This completes the proof of Theorem 3.1.

We next find a sufficient condition for which the integral operator $I$ maps $K_{H}^{0}$ into $S_{H}^{*}(\alpha)$.

Theorem 3.2. If $m_{j}>0$ for $j=1,2$. If for some $\alpha(0 \leq \alpha<1)$, the inequality

$$
\begin{aligned}
m_{1}+m_{2} & +(2-\alpha)\left(1-e^{-m_{1}}\right)+\alpha\left(1-e^{-m_{2}}\right)-\frac{\alpha}{m_{1}}\left(1-e^{-m_{1}}-m_{1} e^{-m_{1}}\right) \\
& -\frac{\alpha}{m_{2}}\left(1-e^{-m_{2}}-m_{2} e^{-m_{2}}\right) \leq 2(1-\alpha)
\end{aligned}
$$

is satisfied, then

$$
I\left(K_{H}^{0}\right) \subset S_{H}^{*}(\alpha) .
$$


Proof. Let $f=h+\bar{g} \in K_{H}^{0}$ where $h$ and $g$ are given by (2) with $B_{1}=0$. We need to prove that $I(f)=H+\bar{G} \in S_{H}^{*}(\alpha)$ where $H$ and $G$ are given by (7) with $B_{1}=0$ are analytic functions in $U$. In view of Lemma 2.2, it is enough to show that $P_{2} \leq 1-\alpha$, where

$$
P_{2}=\sum_{n=2}^{\infty}(n-\alpha)\left|\frac{e^{-m_{1}} m_{1}^{n-1}}{n !} A_{n}\right|+\sum_{n=2}^{\infty}(n+\alpha)\left|\frac{e^{-m_{2}} m_{2}^{n-1}}{n !} B_{n}\right| .
$$

Applying Lemma 2.1, we have

$$
\begin{aligned}
P_{2} & \leq \frac{1}{2}\left[\sum_{n=2}^{\infty}(n-\alpha)(n+1) \frac{e^{-m_{1}} m_{1}^{n-1}}{n !}+\sum_{n=2}^{\infty}(n+\alpha)(n-1) \frac{e^{-m_{2}} m_{2}^{n-1}}{n !}\right] \\
& =\frac{1}{2}\left[\sum_{n=2}^{\infty}\{n(n-1)+n(2-\alpha)-\alpha\} \frac{e^{-m_{1}} m_{1}^{n-1}}{n !}+\right. \\
& \left.\sum_{n=2}^{\infty}\{n(n-1)+n \alpha-\alpha\} \frac{e^{-m_{2}} m_{2}^{n-1}}{n !}\right] \\
& =\frac{1}{2}\left[\sum_{n=2}^{\infty} \frac{e^{-m_{1}} m_{1}^{n-1}}{(n-2) !}+(2-\alpha) \sum_{n=2}^{\infty} \frac{e^{-m_{1}} m_{1}^{n-1}}{(n-1) !}-\alpha \sum_{n=2}^{\infty} \frac{e^{-m_{1}} m_{1}^{n-1}}{n !}\right. \\
& \left.+\sum_{n=2}^{\infty} \frac{e^{-m_{2}} m_{2}^{n-1}}{(n-2) !}+\alpha \sum_{n=2}^{\infty} \frac{e^{-m_{2}} m_{2}^{n-1}}{(n-1) !}-\alpha \sum_{n=2}^{\infty} \frac{e^{-m_{2}} m_{2}^{n-1}}{n !}\right] \\
& =\frac{1}{2}\left[m_{1}+m_{2}+(2-\alpha)\left(1-e^{-m_{1}}\right)+\alpha\left(1-e^{-m_{2}}\right)-\right. \\
& \left.\frac{\alpha}{m_{1}}\left(1-e^{-m_{1}}-m_{1} e^{-m_{1}}\right)-\frac{\alpha}{m_{2}}\left(1-e^{-m_{2}}-m_{2} e^{-m_{2}}\right)\right]
\end{aligned}
$$

The last expression is bounded above by $(1-\alpha)$ by the given hypothesis. Thus the proof of Theorem 3.2 is established.

Theorem 3.3. If $m_{j}>0$, for $(j=1,2)$. If for some $\alpha(0 \leq \alpha<1)$ and the inequality

$$
\begin{aligned}
2 m_{1}^{2} & +(9-2 \alpha) m_{1}+(6-5 \alpha)\left(1-e^{-m_{1}}\right)-\frac{\alpha}{m_{1}}\left(1-e^{-m_{1}}-m_{1} e^{-m_{1}}\right) \\
& +2 m_{2}^{2}+(2 \alpha+3) m_{2}-\alpha\left(1-e^{-m_{2}}\right)-\frac{\alpha}{m_{2}}\left(1-e^{-m_{2}}-m_{2} e^{-m_{2}}\right) \\
& \leq 6(1-\alpha)
\end{aligned}
$$

is satisfied then $I\left(S_{H}^{*, 0}\right) \subset S_{H}^{*}(\alpha)$ and $I\left(C_{H}^{0}\right) \subset S_{H}^{*}(\alpha)$.

Proof. Let $f=h+\bar{g} \in S_{H}^{*, 0}$ where $h$ and $g$ are given by (2) with $B_{1}=0$. We need to prove that $I(f)=H+\bar{G} \in S_{H}^{*}(\alpha)$ where $H$ and $G$ are given by (7) with $B_{1}=0$ are analytic functions in $U$. In view of Lemma 2.2 , it is 
enough to show that $P_{2} \leq 1-\alpha$, where

$$
P_{2}=\sum_{n=2}^{\infty}(n-\alpha)\left|\frac{e^{-m_{1}} m_{1}^{n-1}}{n !} A_{n}\right|+\sum_{n=2}^{\infty}(n+\alpha)\left|\frac{e^{-m_{2}} m_{2}^{n-1}}{n !} B_{n}\right| .
$$

Applying Lemma 2.4, we have

$$
\begin{aligned}
P_{2} & \leq \frac{1}{6}\left[\sum_{n=2}^{\infty}(n-\alpha)(2 n+1)(n+1) \frac{e^{-m_{1}} m_{1}^{n-1}}{n !}+\right. \\
& \left.\sum_{n=2}^{\infty}(n+\alpha)(2 n-1)(n-1) \frac{e^{-m_{2}} m_{2}^{n-1}}{n !}\right] \\
& =\frac{1}{6}\left[\sum_{n=2}^{\infty}\{2 n(n-1)(n-2)+(9-2 \alpha) n(n-1)+(6-5 \alpha) n-\alpha\} \frac{e^{-m_{1}} m_{1}^{n-1}}{n !}\right. \\
& \left.+\sum_{n=2}^{\infty}\{2 n(n-1)(n-2)+(2 \alpha+3) n(n-1)-\alpha n+\alpha\} \frac{e^{-m_{2}} m_{2}^{n-1}}{n !}\right] \\
& =\frac{1}{6}\left[e^{-m_{1}}\left\{2 \sum_{n=2}^{\infty} \frac{m_{1}^{n-1}}{(n-3) !}+(9-2 \alpha) \sum_{n=2}^{\infty} \frac{m_{1}^{n-1}}{(n-2) !}+(6-5 \alpha) \sum_{n=2}^{\infty} \frac{m_{1}^{n-1}}{(n-1) !}-\alpha \sum_{n=2}^{\infty} \frac{m_{1}^{n-1}}{n !}\right\}\right. \\
& \left.+e^{-m_{2}}\left\{2 \sum_{n=2}^{\infty} \frac{m_{2}^{n-1}}{(n-3) !}+(2 \alpha+3) \sum_{n=2}^{\infty} \frac{m_{2}^{n-1}}{(n-2) !}-\alpha \sum_{n=2}^{\infty} \frac{m_{2}^{n-1}}{(n-1) !}+\alpha \sum_{n=2}^{\infty} \frac{m_{2}^{n-1}}{n !}\right\}\right] \\
& =\frac{1}{6}\left[\left\{2 m_{1}^{2}+(9-2 \alpha) m_{1}+(6-5 \alpha)\left(1-e^{-m_{1}}\right)-\frac{\alpha}{m_{1}}\left(1-e^{-m_{1}}-m_{1} e^{-m_{1}}\right)\right\}\right. \\
& \left.+\left\{2 m_{2}^{2}+(2 \alpha+3) m_{2}-\alpha\left(1-e^{-m_{2}}\right)+\frac{\alpha}{m_{2}}\left(1-e^{-m_{2}}-m_{2} e^{-m_{2}}\right)\right\}\right] \\
& \leq 1-\alpha
\end{aligned}
$$

by the given hypothesis.

This completes the proof of Theorem 3.3.

Theorem 3.4. If $m_{j}>0$, for $(j=1,2)$ then $I\left(T S_{H}^{*}(\alpha)\right) \subset T S_{H}^{*}(\alpha)$, if and only if the inequality

$$
\frac{1}{m_{1}}\left(1-e^{-m_{1}}-m_{1} e^{-m_{1}}\right)+\frac{1}{m_{2}}\left(1-e^{-m_{2}}-m_{2} e^{-m_{2}}\right) \leq 1-\frac{1+\alpha}{1-\alpha}\left|B_{1}\right|
$$

is satisfied.

Proof. Let $f=h+\bar{g} \in T S_{H}^{*}(\alpha)$. where $h$ and $g$ are given by (9), we need to prove that the integral operator

$$
I(f)(z)=z-\sum_{n=2}^{\infty} \frac{e^{-m_{1}} m_{1}^{n-1}}{n !}\left|A_{n}\right| z^{n}+\left|B_{1}\right| \bar{z}+\sum_{n=2}^{\infty} \frac{e^{-m_{2}} m_{2}^{n-1}}{n !}\left|B_{n}\right| \overline{z^{n}}
$$

is in $T S_{H}^{*}(\alpha)$, if and only if $P_{3} \leq 1-\alpha$, where

$$
P_{3}=\sum_{n=2}^{\infty}(n-\alpha) \frac{e^{-m_{1}} m_{1}^{n-1}}{n !}\left|A_{n}\right|+(1+\alpha)\left|B_{1}\right|+\sum_{n=2}^{\infty}(n+\alpha) \frac{e^{-m_{2}} m_{2}^{n-1}}{n !}\left|B_{n}\right| .
$$


Using Remark 2.1, we obtain

$$
\begin{aligned}
P_{3} \leq & (1-\alpha)\left[\sum_{n=2}^{\infty} \frac{e^{-m_{1}} m_{1}^{n-1}}{n !}+\sum_{n=1}^{\infty} \frac{e^{-m_{2}} m_{2}^{n-1}}{n !}\right]+(1+\alpha)\left|B_{1}\right| \\
= & (1-\alpha)\left[\frac{1}{m_{1}}\left(1-e^{-m_{1}}-m_{1} e^{-m_{1}}\right)+\frac{1}{m_{2}}\left(1-e^{-m_{2}}-m_{2} e^{-m_{2}}\right)\right] \\
& +(1+\alpha)\left|B_{1}\right| \\
\leq & 1-\alpha
\end{aligned}
$$

by the given condition and this completes the proof of the theorem.

We next explore a sufficient condition which ensures that $I$ maps $K_{H}^{0}$ in to $K_{H}(\alpha)$.

Theorem 3.5. If $m_{j}>0$, for $(j=1,2)$. If for some $\alpha(0 \leq \alpha<1)$, the inequality

$$
e^{m_{1}}\left(m_{1}^{2}+m_{2}^{2}+(4-\alpha) m_{1}+(2-\alpha) m_{2}\right) \leq 2(1-\alpha)
$$

is satisfied then $I\left(K_{H}^{0}\right) \subset K_{H}(\alpha)$.

Proof. Let $f=h+\bar{g} \in K_{H}^{0}$ where $h$ and $g$ are given by (2) with $B_{1}=0$. We need to prove that $I(f)=H+\bar{G} \in K_{H}(\alpha)$ where $H$ and $G$ are given by (7) with $B_{1}=0$ are analytic functions in $U$. In view of Lemma 2.3, it is enough to show that $P_{4} \leq 1-\alpha$, where

$$
P_{4}=\sum_{n=2}^{\infty} n(n-\alpha)\left|\frac{e^{-m_{1}} m_{1}^{n-1}}{n !} A_{n}\right|+\sum_{n=2}^{\infty} n(n+\alpha)\left|\frac{e^{-m_{2}} m_{2}^{n-1}}{n !} B_{n}\right| .
$$

Applying Lemma 2.1, we have

$$
\begin{aligned}
P_{2} & \leq \frac{1}{2}\left[\sum_{n=2}^{\infty}(n-\alpha)(n+1) \frac{e^{-m_{1}} m_{1}^{n-1}}{(n-1) !}+\sum_{n=2}^{\infty}(n+\alpha) \frac{e^{-m_{2}} m_{2}^{n-1}}{(n-2) !}\right] \\
& =\frac{1}{2}\left[\sum_{n=2}^{\infty}\{(n-1)(n-2)+(4-\alpha)(n-1)+2(1-\alpha)\} \frac{e^{-m_{1}} m_{1}^{n-1}}{(n-1) !}\right. \\
& \left.+\sum_{n=2}^{\infty}\{(n-2)+(2+\alpha)\} \frac{e^{-m_{2}} m_{2}^{n-1}}{(n-2) !}\right] \\
& =\frac{1}{2}\left[m_{1}^{2}+(4-\alpha) m_{1}+2(1-\alpha)\left(1-e^{-m_{1}}\right)+m_{2}^{2}+(2-\alpha) m_{2}\right] \\
& \leq 1-\alpha
\end{aligned}
$$

by the given hypothesis.

Thus the proof of Theorem 3.5 is established.

The proof of following theorems are similar to previous theorems so we state only the results. 
Theorem 3.6. If $m_{j}>0$ for $(j=1,2)$ then $I\left(T S_{H}^{*}(\alpha)\right) \subset T K_{H}(\alpha)$, if and only if the inequality

$$
e^{-m_{1}}+e^{-m_{2}} \geq 1+\frac{1+\alpha}{1-\alpha}\left|B_{1}\right|
$$

is satisfied.

Theorem 3.7. If $m_{j}>0$ for $(j=1,2)$ then $I\left(T K_{H}(\alpha)\right) \subset T K_{H}(\alpha)$, if and only if the inequality (15) is satisfied.

Theorem 3.8. If $m_{j}>0$ for $(j=1,2)$. If for some $\alpha(0 \leq \alpha<1)$, the inequality

$$
\begin{gathered}
e^{m_{1}}\left[2\left(m_{1}^{3}+m_{2}^{3}\right)+(15-2 \alpha) m_{1}^{2}+3(8-3 \alpha) m_{1}\right. \\
\left.+(2 \alpha+9) m_{2}^{2}+3(2+\alpha) m_{2}\right] \leq 6(1-\alpha)
\end{gathered}
$$

is satisfied then $I\left(S_{H}^{*, 0}\right) \subset K_{H}(\alpha)$ or $I\left(C_{H}^{0}\right) \subset K_{H}(\alpha)$.

\section{ACKNOWLEDGEMENT}

The authors are thankful to the referee for his/her valuable comments and observations which helped in improving the paper.

\section{REFERENCES}

[1] O.P. Ahuja, Planar harmonic univalent and related mappings, J. Inequal. Pure Appl. Math., 6 (4), Art. 122, 1-18, (2005).

[2] O.P. Ahuja, Harmonic starlikeness and convexity of integral operators generated by hypergeometric series, Int. Transform Spec. Funct., 20(8), 629-641, (2009).

[3] Y. Avci, E. Zlotkiewicz, On harmonic univalent mappings, Ann. Univ. Mariae CurieSklodowska Sect., A 44, 1-7, (2009).

[4] J . Clunie, T Sheil-Small, Harmonic univalent functions, Ann. Acad. Sci. Fen. Series A.I. Math., 9, 3-25, (1984).

[5] Deepmala, A Study on Fixed Point Theorems for Nonlinear Contractions and its Applications, Ph.D. Thesis, Pt. Ravishankar Shukla University, Raipur, Chhatisgarh, India, (2014).

[6] P. Duren, Harmonic Mappings in the Plane, Cambridge University Press, Cambridge, (2004).

[7] R.B. Gandhi, Deepmala, V.N. Mishra, Local and global results for modified Szasz Mirakjan operators, Math. Method. Appl. Sci. (2016), DOI: 10.1002/mma.4171.

[8] J.M. Jahangiri, Harmonic functions starlike in the unit disk, J. Math. Anal. Appl., 235, 470-477, (1999).

[9] V.N. Mishra, Some Problems on Approximations of Functions in Banach Spaces, Ph.D. Thesis, Indian Institute of Technology, Roorkee, Uttarakhand, India, (2007). 
[10] L.N. Mishra, On existence and behavior of solutions to some nonlinear integral equations with Applications, Ph.D. Thesis, National Institute of Technology, Silchar, Assam, India, (2017).

[11] V.N. Mishra, K. Khatri, L.N. Mishra, Deepmala, Inverse result in simulta- neous approximation by Baskakov-Durrmeyer-Stancu operators, Journal of Inequalities and Applications 2013, 2013:586. doi:10.1186/1029-242X-2013- 586.

[12] Saurabh Porwal, An application of a Poisson distribution series on certain analytic functions, J. Complex Anal., Vol.(2014), Art. ID 984135, 1-3.

[13] Saurabh Porwal, Harmonic starlikeness and convexity of integral operators generated by generalized Bessel functions, Acta Math. Vietnam., 39 (2014), 337-346.

[14] Saurabh Porwal, Mapping properties of generalized bessel functions on some subclasses of univalent functions, Analele Universitatii Oradea Fasc. Matematica, XX(2) (2013), 51-60.

[15] A.K. Sharma, Saurabh Porwal, K.K. Dixit, Class mappings properties of convolutions involving certain univalent functions associated with hypergeometric functions, Electronic J. Math. Anal. Appl.,1(2), (2013), 326-333.

[16] H. Silverman, Harmonic univalent function with negative coefficients, J. Math. Anal. Appl., 220, 283-289, (1998).

[17] H. Silverman, E.M. Silvia, Harmonic univalent function with negative coefficients, J. Math. Anal. Appl., 220, 283-289, (1998).

[18] Divesh Srivastava, Saurabh Porwal, Some sufficient conditions for Poisson distribution series associated with conic regions, Int. J. Advanced Technology in Enginering Sci., 3(1) (2015), 229-236.

Saurabh Porwal

Department of Mathematics

UIET, CSJM University, KanPur-208024, (U.P.)

INDIA

E-mail address: saurabhjcb@rediffmail.com

Divesh Srivastava

Department of Mathematics

INTEGRAL UNIVERSiTy, LuCKNOW, (U.P.)

INDIA

E-mail address: divesh2712@gmail.com 